\title{
Ganciclovir Ophthalmic Gel in the Treatment of Herpes Simplex Keratitis
}

\author{
Penny A Asbell, MD, FACS, MBA \\ Professor of Ophthalmology, Director of Cornea and Refractive Services, Department of Ophthalmology, Mount Sinai School of Medicine
}

\begin{abstract}
Herpes simplex keratitis (HSK) is the most common form of viral keratitis and is a common cause of ocular morbidity and blindness. HSK is caused by the herpes simplex virus (HSV) and occurs in patients with no previous exposure to HSV typically occurring during childhood, or in patients with latent disease bearing dormant virus in the nerve ganglia or the cornea. HSK initially presents as a cluster of small, clear vesicles in the epithelium that accumulates to form dendritic lesions which can progress to geographic ulcers, whilst further progression of infection deeper into the cornea affects the stroma. Ganciclovir (GCV) was developed for the treatment of acute superficial herpetic keratitis as a replacement for earlier, less effective, or less tolerated antiviral therapy. Studies have found topical GCV gel treatment to be safe and well tolerated with more favorable pharmacokinetics than acyclovir. The recent approval by the US Food and Drug Administration (FDA) for the clinical use of $0.15 \%$ GCV gel for treatment of HSK will provide patients and physicians with a more tolerable, convenient, and effective treatment.
\end{abstract}

\section{Keywords}

Acyclovir, adenoviral keratoconjunctivitis, ganciclovir, herpes simplex keratitis, ocular herpes, ophthalmic gel

Disclosure: Penny A Asbell, MD, FACS, MBA, has acted as a consultant for Alcon, Aton, Bausch \& Lomb, Inspire, Johnson \& Johnson, Merck, Pfizer, Santen, and Vistakon Pharma, has received research funding from the National Institutes of Health, Research to Prevent Blindness, the Toni and Martin Sosnoff Fund, Bausch \& Lomb, Alcon, and Inspire, and has received educational grants from Santen and Inspire.

Acknowledgment: Editorial assistance was provided by Touch Briefings.

Received: January 21, 2011 Accepted: March 1, 2011 Citation: US Ophthalmic Review, 2011;4(1):63-8 DOl: 10.17925/USOR.2011.04.01.63

Correspondence: Penny A Asbell, MD, FACS, MBA, Professor of Ophthalmology, Director of Cornea and Refractive Services, Department of Ophthalmology, Mount Sinai School of Medicine, 1190 Fifth Avenue, New York, New York 10029. E: penny.asbell@mssm.edu

Support: The publication of this article was funded by Bausch \& Lomb. The views and opinions expressed are those of the author and not necessarily those of Bausch \& Lomb.

\section{Herpes Simplex Keratitis-A Significant Burden and Threat to Human Eyesight}

Herpes simplex keratitis (HSK), caused by the herpes simplex virus (HSV), is the most common form of viral keratitis and is a common cause of ocular morbidity and blindness. ${ }^{1.2}$ HSV infection is extremely common in populations worldwide. It is one of the more common severe infectious agents of the fetal and newborn periods, usually occurring after exposure to the virus during delivery. ${ }^{3}$ Although infection is usually acquired in early childhood, increasing age correlates with a significant increase in the percentage of the population that is seropositive..$^{4-6} \mathrm{In}$ the US, nearly $60 \%$ of the population has shown evidence of infection with nearly 20,000 new primary cases of ocular herpes per year?

Approximately $1 \%$ of infected persons develop ocular outbreaks. ${ }^{8}$ Ocular disease may be one of two forms-primary disease in patients with no previous exposure to HSV typically occurring during childhood, or latent disease in patients bearing dormant virus in the nerve ganglia or the cornea which can be reactivated by factors such as stress, ultraviolet (UV) radiation, and menstruation. ${ }^{9.10}$ Recurrent disease is more common, accounting for 30,000 of the 50,000 cases of HSK diagnosed each year. ${ }^{1,12}$ Lesions are also common in immunosuppressed individuals, such as recent organ transplant patients or patients with human immunodeficiency virus (HIV). ${ }^{8}$ HSV type 1 is almost exclusively responsible for ocular and orofacial infections whilst HSV type 2 generally causes genital disease. HSV type 2 may infect the eye by means of orofacial contact with genital lesions and is occasionally transmitted to neonates at birth when the mother has a genital HSV type 2 infection.

HSK initially presents as a cluster of small, clear vesicles in the epithelium that accumulate to form dendritic lesions (see Figure 1), occurring in approximately $15 \%$ of initial episodes of ocular HSV. ${ }^{13}$ Left untreated, they can progress to geographic ulcers, a type of large epithelial defect with fimbriated edges. With progression of infection deeper into the cornea, the stroma may become affected-stromal keratitis represents approximately $2 \%$ of primary ocular HSV-1 infections. ${ }^{14}$

Patients may have latent HSV in the trigeminal ganglion or cornea, and recurrent episodes of ocular disease can increase in incidence over time, leading to an elevated risk of corneal damage and blindness. ${ }^{2,15}$ Patients with severe corneal damage are candidates for corneal transplantation, but recurrence of HSK in the corneal graft may result in structural damage leading to graft rejection and failure. ${ }^{2,16}$ Upon 
Figure 1: Fluorescein-stained Dendritic (A) and Geographic (B) Corneal Ulcers Caused by Herpes Simplex Viral Infection ${ }^{8}$
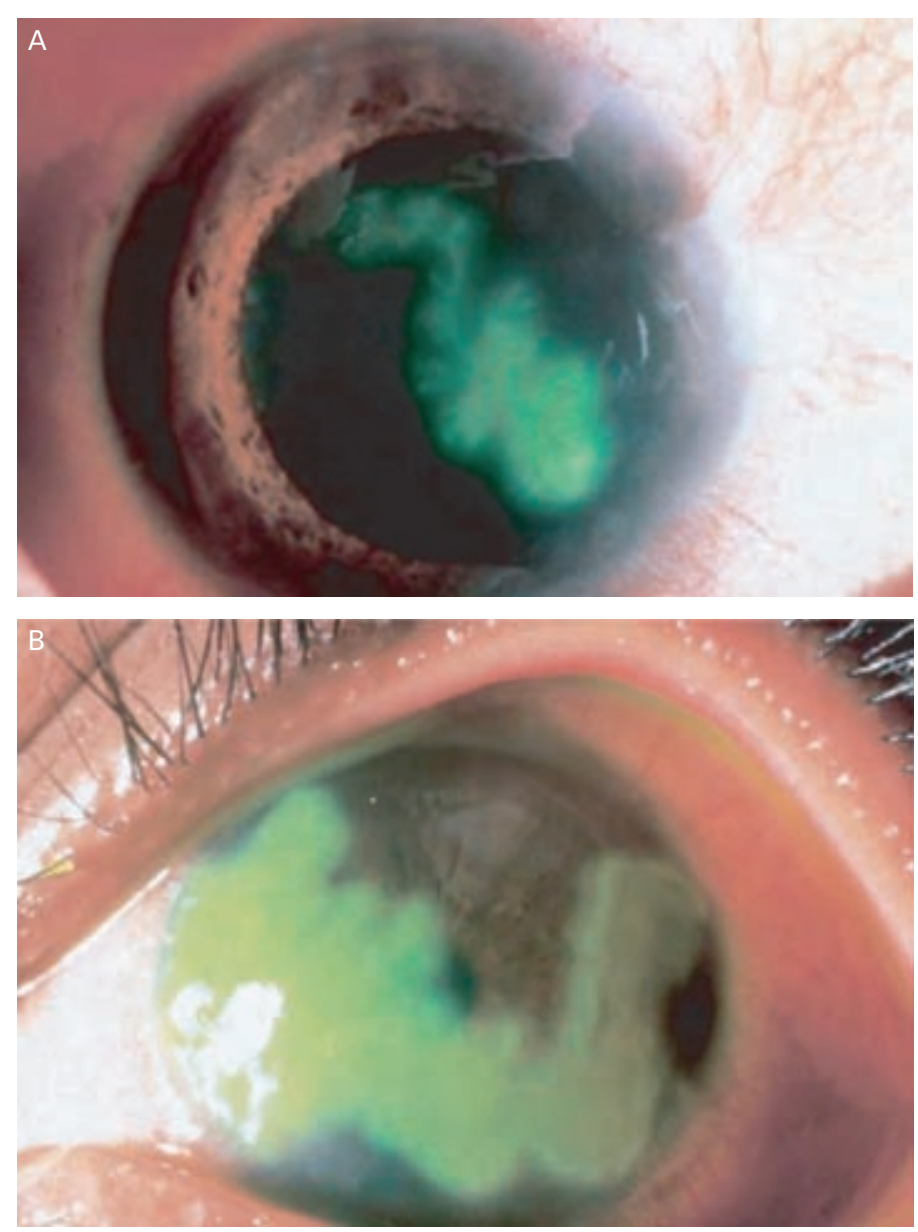

diagnosis, immediate treatment of HSK is necessary if complications leading to blindness are to be limited. ${ }^{8}$ This article will discuss the treatments approved for HSK and in particular the evidence supporting the use of ganciclovir (GCV) for this indication.

\section{Treatments for Herpes Simplex Keratitis}

Epithelial dendritic keratitis is usually treated with oral and/or topical antiviral therapy. ${ }^{8}$ The antiviral agent trifluridine (TFT), approved in 1980, is available in the US and Canada as a $1 \%$ ophthalmic solution (Viroptic ${ }^{\circledR}$ ). It is a nucleoside analogue, a modified form of deoxyuridine, which results in the inhibition of DNA synthesis of both cells infected with HSV and healthy epithelial cells. This non-specificity can lead to epithelial toxicity and further damage to the cornea., ${ }^{8,17-20}$ Other topically applied ophthalmic antivirals that have been used in Europe and the US include idoxuridine, iododesoxycytidine, and vidarabine. However, due to high toxicity their use has been superseded in favor of more selective agents. ${ }^{8,12,17}$ Acyclovir (ACV) and GCV are two more recently developed antivirals with improved selectivity and decreased toxicity compared with their predecessors. ${ }^{8}$

Outside the US, ACV ophthalmic ointment (3\%) and GCV ophthalmic gel (0.15\%) are the European standard of care for first-line treatment of acute superficial HSK. ACV has been used in HSK therapy for several years and has proven efficacy in both clinical trials and in clinical practice. ${ }^{8,17,21-24}$ ACV ointment or TFT combined with topical interferon produced improved treatment success compared to use of the nucleoside antiviral agent alone. ${ }^{25-28} \mathrm{GCV}$ is an effective and well-tolerated treatment for HSK with more favorable pharmacokinetics than ACV. Since 1995, the $0.15 \%$ gel preparation of GCV has been commercially available in 30 countries within Europe, Asia, Africa, and South America. In 2007, it was granted orphan drug designation for treatment of HSK, and in 2009, it gained Food and Drug Administration (FDA) approval for this indication. ${ }^{17} \mathrm{GCV}$ in gel form causes less blurring than ACV petrolatum-based ointment. 2,8,17,22,29,30 ACV and GCV have been widely used to treat herpetic keratitis with viral resistance typically isolated to patients that are immunocompromised. ${ }^{31,32}$

Other treatments include debridement, which has some success particularly when used in combination with antiviral treatments. ${ }^{12}$ Following medical therapy, complications of herpes keratitis, such as corneal scarring, may require additional surgical management. Irregular astigmatism resulting from chronic stromal keratitis may be corrected with the use of rigid gas-permeable contact lenses. ${ }^{33}$ Penetrating keratoplasty can be used for visual rehabilitation of patients with corneal opacities or perforations. ${ }^{34} \mathrm{~A}$ small perforation in an inflamed eye may be managed with a tissue adhesive, bandage contact lens, and/or amniotic membrane transplantation. ${ }^{35,36}$

\section{Mode of Action of Ganciclovir and Other Treatments for Herpes Simplex Keratitis}

GCV is a nucleoside analog of deoxyguanosine. In infected cells, GCV is phosphorylated by viral thymidine kinase (VTK). ${ }^{37,38}$ Cellular TK has a low affinity for GCV because the deoxyribose mimicking moiety inhibits enzyme binding..$^{39,40} \mathrm{GCV}$ in therapeutic doses freely moves into and out of uninfected cells. However, once phosphorylated GCV is trapped within the cells due to its increased polarity. ${ }^{37}$ Thus, GCV accumulates in virus-infected cells. GCV monophosphate is then recognized by cellular kinases which form di- and triphosphoganciclovir (GCV-TP). GCVTP inhibits viral DNA replication in two ways. Firstly, GCV-TP competitively inhibits deoxyguanosine triphosphate incorporation into DNA, slowing viral DNA synthesis. Secondly, incorporation of GCV results in DNA chain termination-additional nucleotides cannot be added on as GCV lacks the essential deoxyribose..$^{40,41}$ As uninfected healthy tissues are largely unaffected, the toxicity of the treatment is low (see Figure 2)., 2,17,42

ACV has a similar mechanism of action to GCV, undergoing phosphorylation by VTK, leading to DNA chain termination. ${ }^{42}$ Intracellular GCV-TP concentrations are 10-fold higher compared to ACV-TP and its decline is much slower with an intracellular half-life exceeding 24 hours. ${ }^{37,43,44}$ Furthermore, HSV mutants that are resistant to ACV due to mutated VTK or DNA polymerase remain susceptible to GCV treatment. ${ }^{45}$

Both ACV and GCV are more selective than TFT, which is phosphorylated in infected as well as in non-infected cells, and serves as a substrate for both host and DNA polymerases. ${ }^{46,8}$ Thus, TFT inhibits viral replication but healthy tissues are affected, rendering it highly toxic. ${ }^{20}$ In the eye, the toxicity can manifest as ocular epithelial toxicity, resulting in delayed healing of the corneal epithelium and stroma, corneal epithelial dysplasia, or conjunctival scarring. $8,17,20,46$ 


\section{Clinical Trials Evidence for the Use of Ganciclovir in the Treatment of Herpes Simplex Keratitis}

Preclinical studies of GCV gel have demonstrated effective treatment of herpetic keratitis. Experimental models of herpetic keratitis in rabbits and mice have shown treatment with GCV 0.15\% prevented lesion formation and had greater efficacy than ACV. ${ }^{47-50}$ Additionally, chronic administration of GCV in rabbits with HSK resulted in clinical efficacy and a significant reduction of corneal ulcer area, clouding, and vascularization. ${ }^{51}$

GCV was developed for the treatment of acute superficial herpetic keratitis as a replacement for earlier, less effective, or less tolerated antivirals. Studies have found topical GCV gel treatment to be safe and well tolerated. ${ }^{29}$ Two further clinical trials in France examined GCV ophthalmic gel in randomized, double-masked studies evaluating pharmacokinetics and tolerance in healthy volunteers. All volunteers completed the studies and reported good tolerance and no significant discomfort with only mild anomalies of no clinical consequence following the application of the GCV gel. ${ }^{8}$

Phase II/III trials of GCV gel all occurred in treatment centers outside North America and have not included any patients with HSK from US centers. Clinical studies conducted in Europe, Asia, and Africa involving over 370 patients, comparing the efficacy and tolerability of GCV ophthalmic gel with ACV ointment in herpetic keratitis, showed that, although not always statistically significant, GCV-treated patients tended to have higher overall healing rates accompanied with lower relapse rates than the ACV-treated patients (see Table 1, Figures 3 and 4). ${ }^{8,22,29}$

The first randomized, prospective phase II clinical trials occurred in the early 1990s, involving 66 patients from across Africa and a further 37 patients from Europe. Patients with herpetic keratitis were treated with either GCV $(0.15 \%, 0.05 \%)$ gel or ACV (3\%) petrolatum-based ointment five times daily until healing and then three times daily for one week. In the African study, the rate of healing was $82.6 \%$ for GCV $0.15 \%, 77.3 \%$ for GCV $0.05 \%$, and $72.7 \%$ ACV $3 \%$, whilst the median healing time was seven days for the GCV treated patients and eight days for the ACV patients. Greater tolerance and compliance were observed, accompanied by less stinging and burning sensations in the GCV treated groups compared to the ACV treated groups. ${ }^{29}$ In the European study, patients experienced less blurring in the GCV groups compared to ACV treatment (38.5\% and $76.9 \%$, respectively), while less burning/stinging sensations were reported by patients receiving the GCV $(0.15 \%$ and $0.05 \%)$ treatment compared to ACV (16.7\% and 50\%, respectively). ${ }^{29} \mathrm{~A}$ third clinical study undertaken in Karachi, Pakistan, involved 109 patients in a similar randomized, controlled trial design as the European and African trials. Similar to the earlier trials, this study reported GCV to have generally improved healing rates, and lower incidences of relapse and withdrawal when compared to ACV treatment. ${ }^{8}$ The elevated withdrawal rate in the ACV treated patients was due to treatment failure relating to worsening condition or reduced therapeutic efficacy.

There has been one large multicenter, prospective, randomized phase III study examining the effects of topical GCV gel and ACV ointment for the treatment of HSV herpetic keratitis. ${ }^{22}$ This study involved 164 patients across 28 study centers within Europe. Analysis of the clinical study results indicate that GCV $0.15 \%$ was at least as effective as ACV $3 \%$ for
Figure 2: Trifluridine and Ganciclovir Mechanism of Action

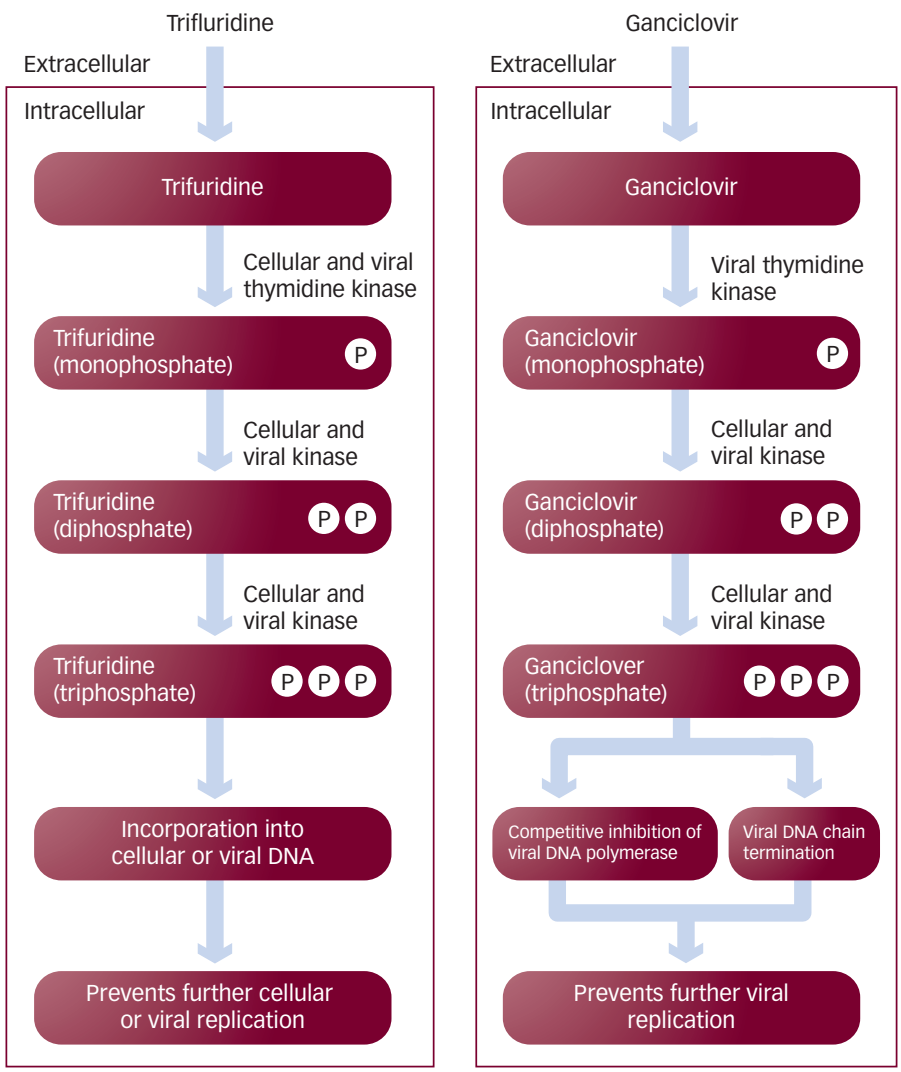

$P=$ phosphate. Source: Zirgan monograph, Bausch \& Lomb 2010

treating acute herpetic keratitis with similar median time to healing. Patients treated with GCV did however report less blurring and fewer incidences of stinging or burning sensations. ${ }^{8,22}$

GCV can penetrate the corneal stroma following topical application which results in therapeutic levels of GCV in the aqueous humor. Results from the previous clinical studies demonstrate that GCV is as effective as ACV for treating herpetic keratitis in humans, and, when applied in gel form, causes less blurring of vision than the petrolatum-based ointment applications, leading to greater tolerance of the treatment and potentially, to better compliance. A further non-randomized clinical study examined the potential efficacy of GCV for treating patients with herpetic keratitis. ${ }^{52}$ Complete resolution of herpetic keratitis was noted in all patients. The six patients on prophylactic GCV did not develop recurrence of herpetic keratitis, including three patients who had undergone penetrating keratoplasty. ${ }^{52}$ The lack of clinical controls or masking notwithstanding, this study demonstrates the potential value of GCV in the treatment and prophylaxis of HSV herpetic keratitis, including in those patients that have undergone corneal grafts.

\section{Ganciclovir in Clinical Use for Viral Keratitis Treatment}

The commercial availability of GCV 0.15\% gel across Europe, Asia, Africa, and South America has led to a considerable number of patients with herpes infections being treated by physicians. The proportion of cases where the investigator judged the tolerance to the product as excellent 
Table 1: Key Studies Supporting the Use of Ganciclovir in Herpes Simplex Keratitis

\begin{tabular}{|c|c|c|}
\hline Study Type & $\begin{array}{l}\text { Number of Patients } \\
\text { and Centers }\end{array}$ & $\begin{array}{l}\text { Treatments-GCV } \\
\text { and Comparator }\end{array}$ \\
\hline $\begin{array}{l}\text { Phase II parallel group, } \\
\text { randomized, comparative, } \\
\text { multicenter study }\end{array}$ & $\begin{array}{l}67 \text { pts } \geq 12 \text { years of age } \\
\text { with HSK at four centers } \\
\text { in Africa Dendritic ( } 51 \text { ) } \\
\text { or geographic (16) } \\
\text { epithelial keratitis }\end{array}$ & $\begin{array}{l}\text { Pts treated with } 0.15 \% \text { GCV } \\
\text { gel }(n=23), 0.05 \% \text { GCV ( } n=22) \text {, } \\
\text { or } 3 \% \text { ACV ( } n=22) \text { five times } \\
\text { daily until ulcer healed then } \\
\text { three times daily for one week }\end{array}$ \\
\hline
\end{tabular}

Efficacy Findings

For GCV 0.15\%, GCV 0.05\%,

and ACV 3\%, healing rates

were $82.6,77.3$, and $72.7 \%$.

Investigator assessments of

efficacy as very satisfactory

(on day 14)—71.43, 66.67,

and $52.63 \%$ (ns).

Conjunctival samples showed

an identical rate of viral

elimination in GCV $0.15 \%$ and

ACV $3 \%$, but GCV $0.05 \%$ was

less effective

Phase II parallel group

randomized, comparative,

multicenter study

For GCV 0.15\% and ACV 3\%,

respectively, healing rates

were 83.3 and $70.6 \%$ (ns).

\section{7 pts $\geq 18$ years with HSK}

at four centers in France.

Dendritic (36) or geographic

(one) epithelial keratitis
Pts treated with $0.15 \% \mathrm{GCV}$

$(n=19)$ or ACV $3 \%(n=18)$ five times daily until ulcer healed then three times daily for one week
Withdrawals due to worsening

ulcer or complications were

11.1 and $41.2 \%(p=0.06)$.

Median time of healing was six

days for GCV $0.15 \%$ and seven

days for ACV 3\% ( $p=0.056)$

For GCV 0.15\%, GCV 0.05\%,

and ACV $3 \%$, respectively,

healing rates were 86.1 ,

80 , and $71.1 \%$ (ns).

Withdrawals due to

complications were 5.6,

11.4, and $21.05 \%(p=0.02)$

Stromal damage in withdrawals

occurred in 50,50, and

$62.5 \%$ of pts

Phase III parallel group

randomized, comparative,

multicenter study
164 pts at 28 centers in

Europe. Dendritic (138) or

or geographic (26)

epithelial keratitis
Pts treated with $0.15 \% \mathrm{GCV}$

$(n=84)$, or ACV $3 \%(n=80)$, five

times daily until ulcer healed

then three times daily for

one week
Dendritic ulcers ( $n=137)$ and

geographic ulcers $(n=27)$

were reported separately. In

dendritic ulcers treated with

GCV $0.15 \%$ and ACV 3\%,

respectively, healing rates

were 88.7 and $91 \%$ (ns).

Both groups showed similar

rates of withdrawals (ns)
Safety Findings

GCV 0.15 and $0.05 \%$ were well tolerated-stinging and burning sensations were less common than ACV $3 \%(p=0.10)$. Rates of toxic superficial punctate keratitis were similar in all three groups (ns) $)^{8,29}$

\section{For GCV 0.15\% and ACV 3\% \\ groups 38.5 and $76.9 \%$ of \\ pts experienced blurred vision \\ for $>5$ minutes. For GCV $0.15 \%$ \\ $100 \%$ of investigators and $100 \%$ \\ of pts rated general tolerance as \\ 'excellent'. For ACV 3\% these \\ were 75 and $67 \%$ 8,29 \\ Tolerability was acceptable for all three groups. The incidence of superficial punctate keratitis cases appearing or exacerbated by treatment was similar for all three groups. No hematological effects observed ${ }^{8}$}

At Day 14 , fewer subjects in the
GCV $0.15 \%$ group reported a
stinging or burning sensation
than in the ACV $3 \%$ group
( $p=0.03$ ). The rate of punctate
keratitis was lower in the GCV
$0.15 \%$ group. The proportions
of pts ( $p=0.0002$ ) and
investigators ( $p=0.00006$ )
rating the tolerance to the
product as excellent were
significantly greater for the GCV
$0.15 \%$ group than the ACV
$3 \%$ group ${ }^{8,22}$

$A C V=$ acyclovir; $G C V=$ ganciclovir; $H S K=$ herpes simplex keratitis; $P$ Pts = patients; $n s=$ non significant.

was significantly higher in the GCV group (78.7\%) compared with the ACV group (43.94\%) treated for dendritic ulcers. Similarly, patients reported that the overall tolerance with GCV was more frequently considered excellent $(75.36 \%)$ compared with ACV $(42.42 \%) .{ }^{8}$ This may result in a greater level of compliance amongst patients.

There is discussion amongst physicians that GCV $0.15 \%$ gel could be suitable for eyelid application/nasal ulcerations. However, in the US, this recommendation is not included in the package insert, and is therefore described as off-label, non-FDA approved use. However, since GCV gel is approved for ocular use it is likely to be safe for use on eyelids or for nasal ulcerations. The use of GCV for this indication has not yet been clinically tested and the FDA does not give approval unless there are clinical trial data in support of a treatment. Currently there are no clinical studies which address whether $0.15 \%$ GCV gel can be used for the prophylactic management of corneal ulcerations. However, the Herpetic Eye Disease study (HEDS) to investigate the effect of oral acyclovir therapy for recurrences of HSV epithelial keratitis and stromal keratitis 
found that the overall near $50 \%$ lower risk of any type of recurrence in the systemic acyclovir group was also generally observed in subgroups of patients based on other baseline factors, such as time since last episode of ocular HSV, age, history of non-ocular disease, and prior ocular HSV history. ${ }^{53}$ Whether a similar effect with topical use of GCV would be achieved has not been studied and further clinical data are required..$^{53}$ Reactivation of HSV and recurrence of herpetic keratitis in patients that have received a corneal transplant may lead to corneal graft rejection and blindness. Therefore, long-term prophylaxis with topical GCV $0.15 \%$ gel might help to prevent recurrence of the disease and subsequent graft rejection. The use of GCV as a prophylactic agent appears to be a good therapeutic strategy, however dosing and frequency still remain to be determined. ${ }^{22,30,52}$

\section{Ganciclovir in the Treatment of Other Ocular Infections}

GCV $0.15 \%$ gel has been shown to have therapeutic potential in the treatment of a number of other ocular infections. Several in vitro and in vivo studies have examined the use of GCV in the treatment of adenoviral keratoconjunctivitis. GCV was observed to reduce the incidence and duration of viral infection compared to placebo treatment. In a study of the $50 \%$ effective dose (ED50) for known adenoviral serotypes and one adenoviral clinical isolate, the resulting ED50s ranged from 4.5 to $33 \mu \mathrm{M} .{ }^{54,55}$ Two small clinical trials have also demonstrated the efficacy of GCV $0.15 \%$ in treating adenoviral keratoconjunctivitis. GCV treatment resulted in quicker recovery time and fewer subepithelial opacities compared to placebo. In the open-label uncontrolled study, ocular discomfort was relieved in one week and no patients developed keratitis. ${ }^{56,57}$ Adenoviral infections, commonly causing patient morbidity and chronic visual disturbances associated with adenovirus keratoconjunctivitis, have been controlled and treated with GCV $0.15 \%$ gel..$^{39,54,58-61}$ There are in vitro studies that demonstrate GCV is a powerful inhibitor of Varicella Zoster virus (VZV) replication. ${ }^{40} \mathrm{GCV}$ either in combination or alone has been used to treat progressive outer retinal necrosis due to VZV, however there are no published studies on the use of GCV in the treatment of keratitis due to VZV. GCV (Vitrasert) has also been used to beneficially treat sight-threatening cytomegalovirus (CMV) retinitis in immunocompromised and HIV patients. ${ }^{62,63}$ Further off-label indications include the management of acute retinal necrosis. ${ }^{64-66}$

\section{Future Developments in Ganciclovir for Keratitis Treatment}

Topical GCV $0.15 \%$ gel has been shown to be as effective as ACV in the treatment of active HSK. ${ }^{8}$ Recurrent herpetic keratitis can result in extensive damage to the cornea and blindness, and recurrence in a corneal graft can result in graft rejection or failure. Prophylaxis with topical GCV may reduce the incidence of graft rejection or damage following recurrence of the disease. A recent prospective study examined the use of topical GCV $0.15 \%$ for prophylactic treatment of herpetic keratitis. Complete resolution in the patients was noted and none of the patients developed a recurrence of the herpetic keratitis during the follow-up period. ${ }^{52}$ However, the utility of GCV as a prophylactic agent remains to be determined, with doses and frequency still unclear. Furthermore, the intraocular penetration of GCV into the aqueous humor makes it a potential candidate for the treatment and prevention of herpetic keratouveitis, HSV, and CMV anterior uveitis. ${ }^{2}$ As such, further prospective, randomized,
Figure 3: Recovery at Day 14 in Four Studies Comparing Ganciclovir with Acyclovir for the Treatment of Herpes Simplex Keratitis

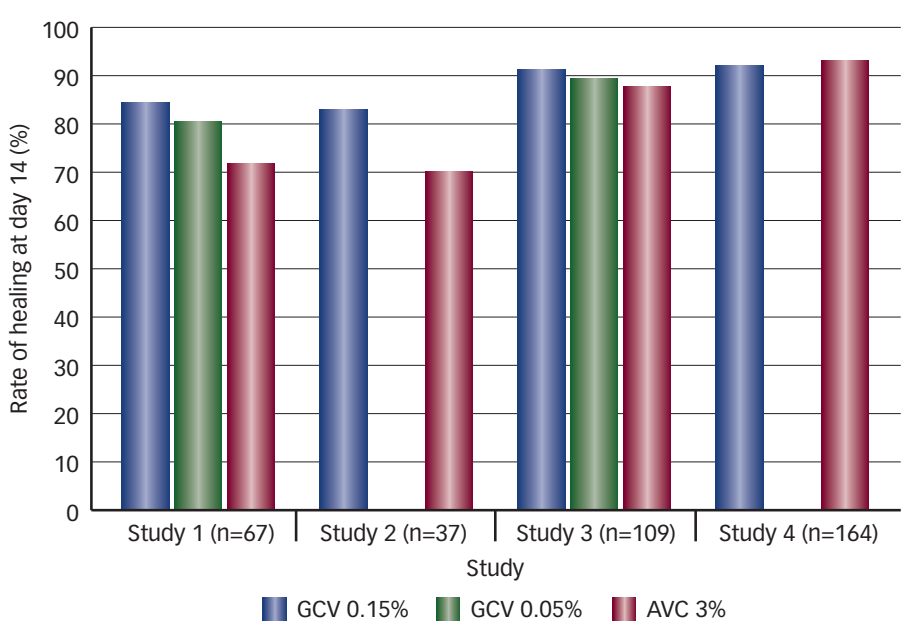

Data for per protocol populations are given for each study. Studies two and four did not include a GCV $0.05 \%$ treatment arm. Differences between ganciclovir (GCV) and acyclovir (ACV) in all four studies were non-significant.

Figure 4: Proportion of Patients Withdrawing from Treatment Due to Worsening Condition or Complications in Four Studies Comparing Ganciclovir with Acyclovir for the Treatment of Herpes Simplex Keratitis

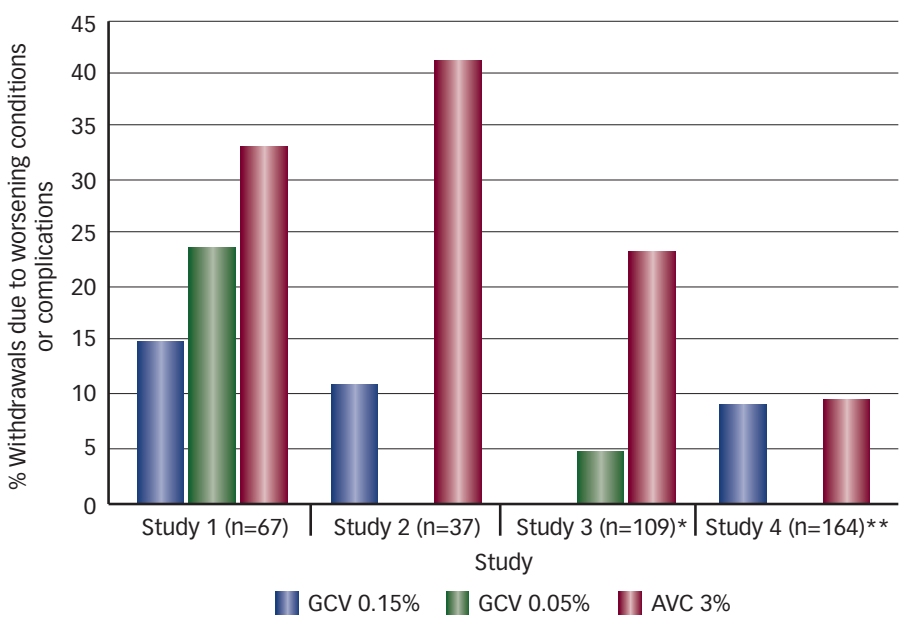

Data for per protocol populations are given for each study. Studies two and four did not include a GCV $0.05 \%$ treatment arm. *Differences between ganciclovir (GCV) and acyclovir $(\mathrm{ACV})$ were significant in study three in the per-protocol analysis only $(p=0.02)$, in studies one, two, and four these differences were non-significant. **Data for study four are for dendritic ulcers only.

controlled multicenter clinical trials are required in the future to asses the long-term safety and efficacy of topical GCV application.

The development of more effective antiviral treatments has significantly improved the prognosis for patients with HSK. Trifluridine has been the only approved and marketed treatment in the US. However, there are concerns regarding its non-specific action and toxic side effects. The recent approval by the FDA for the $0.15 \%$ GCV gel in the clinical use for treatment of HSK will provide patients and physicians with a more tolerable, convenient, and effective treatment. 


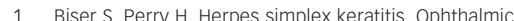
Hyperguide: Corneal and Anterior Segment Diseases, 2007. Available at: www.ophthalmic.hyperguides.com (accessed January 6, 2011).

2. Tabbara KF, Al Balushi N, Topical ganciclovir in the treatment of acute herpetic keratitis, Clin Ophthalmol, 2010;4:905-12.

3. Muller WJ, Jones CA, Koelle DM, Immunobiology of herpes simplex virus and cytomegalovirus infections of the fetus and newborn, Curr Immunol Rev, 2010;6:38-55.

4. Isacsohn M, Smetana Z, Rones ZZ, et al., A sero-epidemiological study of herpes virus type 1 and 2 infection in Israel, J Clin Virol, 2002;24:85-92.

5. Smith JS, Robinson NJ, Age-specific prevalence of infection with herpes simplex virus types 2 and 1: a global review, I Infect Dis, 2002;186(Suppl. 1):S3-28.

6. Xu F, Schillinger JA, Sternberg MR, et al., Seroprevalence and coinfection with herpes simplex virus type 1 and type 2 in the United States, 1988-1994, J Infect Dis, 2002;185:1019-24.

7. TJ, Epidemiology and natural history of ocular herpes simplex virus infection in Rochester, Minnesota, 1950-1982 Trans Am Ophthalmol Soc, 1988;86:688-724.

8. Colin J, Ganciclovir ophthalmic gel, $0.15 \%$ : a valuable tool for treating ocular herpes, Clin Ophthalmol, 2007;1:441-53.

9. El Hayderi L, Raty L, Failla V, et al., Severe herpes simplex virus type-l infections after dental procedures, Med Oral Patol Oral Cir Bucal, 2011:16:e15-8.

10. Elftman MD, Hunzeker JT, Mellinger JC, et al., Stress-induced glucocorticoids at the earliest stages of herpes simplex virus-1 infection suppress subsequent antiviral immunity, implicating impaired dendritic cell function, J Immunol, 2010;184:1867-75.

11. Liesegang TJ, Melton LJ, 3rd, Daly PJ, et al., Epidemiology of ocular herpes simplex. Incidence in Rochester, Minn, 1950 through 1982, Arch Ophthalmol, 1989;107:1155-9.

12. Wilhelmus KR, The treatment of herpes simplex virus epithelial keratitis, Trans Am Ophthalmol Soc, 2000;98:505-32.

13. Darougar S, Wishart MS, Viswalingam ND, Epidemiological and clinical features of primary herpes simplex virus ocular infection, Br J Ophthalmol, 1985;69:2-6.

14. Liesegang TJ, Herpes simplex virus epidemiology and ocular importance, Cornea, 2001;20:1-13

15. Wilhelmus KR, Therapeutic interventions for herpes simplex virus epithelial keratitis, Cochrane Database Syst Rev, 2007; CD002898.

16. De Kesel RJ, Koppen C, leven M, et al., Primary graft failure caused by herpes simplex virus type 1, Cornea, 2001:20:187-90.

17. Kaufman $\mathrm{H}$, Ganciclovir: a promising topical antiviral gel for herpetic keratitis, Expert Review of Ophthalmology, 2009;4:367-75.

18. Ia Lau C, Oosterhuis JA, Versteeg J, et al., Aciclovir and trifluorothymidine in herpetic keratitis. Preliminary report of a multicentered trial, Doc Ophthalmol, 1981:50:287-90.

19. Majumdar S, Tirucherai GS, Pal D, et al., Functional differences in nucleoside and nucleobase transporters expressed on the rabbit corneal epithelial cell line (SIRC) and isolated rabbit cornea, AAPS PharmSci, 2003;5:E15.

20. Maudgal PC, Van Damme B, Missotten L, Corneal epithelial dysplasia after trifluridine use, Graefes Arch Clin Exp Ophthalmol, 1983;220:6-12.

21. Au E, Sacks SL, Therapeutic Options for Herpes Simplex Infections, Curr Infect Dis Rep, 2003:5:22-7.

22. Hoh HB, Hurley C, Claoue C, et al., Randomised trial of ganciclovir and acyclovir in the treatment of herpes simplex dendritic keratitis: a multicentre study, Br J Ophthalmol, 1996;80:140-3.

23. Tilson $\mathrm{HH}$, Engle $\mathrm{CR}$, Andrews EB, Safety of acyclovir: a summary of the first 10 years experience, J Med Virol, 1993;Suppl 1:67-73.

24. Whitley RJ, Roizman B, Herpes simplex virus infections, Lancet 2001:357:1513-8.
25. Cantell K, Development of antiviral therapy with alpha interferons: promises, false hopes and accomplishments, Ann Med, 1995;27:23-8

26. Colin J, Chastel C, Renard G, et al., Combination therapy for dendritic keratitis with human leukocyte interferon and acyclovir, Am J Ophthalmol, 1983:95:346-8.

27. de Koning EW, van Bijsterveld OP, Cantell K, Combination therapy for dendritic keratitis with acyclovir and alphainterferon, Arch Ophthalmol, 1983;101:1866-8.

28. Sundmacher R, Cantell $K$, Mattes A, Combination therapy for dendritic keratitis, High-titer alpha-interferon and trifluridine, Arch Ophthalmol, 1984;102:554-5.

29. Colin J, Hoh HB, Easty DL, et al., Ganciclovir ophthalmic gel (Virgan; $0.15 \%$ ) in the treatment of herpes simplex keratitis, Cornea, 1997;16:393-9.

30. Tabbara KF, Pharmacologic strategies in the prevention and treatment of corneal transplant rejection, Int Ophthalmol, 2008;28:223-32.

31. Greco A, Diaz JJ, Thouvenot D, et al., Novel targets for the development of anti-herpes compounds, Infect Disord Drug Targets, 2007;7:11-8.

32. Levin MJ, Bacon TH, Leary JJ, Resistance of herpes simplex virus infections to nucleoside analogues in HIV-infected patients, Clin Infect Dis, 2004;39 Suppl 5:S248-57.

33. Ozkan B, Elibol O, Yuksel N, et al., Why do patients with improved visual acuity drop out of RGP contact lens use? Ten-year follow-up results in patients with scarred corneas, Eur J Ophthalmol, 2009;19:343-7.

34. Cobo LM, Coster DJ, Rice NS, et al., Prognosis and management of corneal transplantation for herpetic keratitis, Arch Ophthalmol, 1980;98:1755-9.

35. Arentsen J, Laibson PR, Cohen EJ, Management of corneal descemetoceles and perforations, Trans Am Ophthalmol Soc 1984:82:92-105

36. Shi $\mathrm{W}$, Chen $\mathrm{M}, \mathrm{Xie} \mathrm{L}$, Amniotic membrane transplantation combined with antiviral and steroid therapy for herpes necrotizing stromal keratitis, Ophthalmology, 2007;114:1476-81.

37. Cheng YC, Grill SP, Dutschman GE, et al., Metabolism of 9-(1,3dihydroxy-2-propoxymethyl)guanine, a new anti-herpes virus compound, in herpes simplex virus-infected cells, J Biol Chem 1983a;258:12460-4.

38. Naesens L, Lenaerts L, Andrei G, et al., Antiadenovirus activities of several classes of nucleoside and nucleotide analogues, Antimicrob Agents Chemother, 2005;49:1010-6.

39. Freitas VR, Smee DF, Chernow M, et al., Activity of 9-(1,3dihydroxy-2-propoxymethyl)guanine compared with that of acyclovir against human, monkey, and rodent cytomegaloviruses, Antimicrob Agents Chemother, 1985;28:240-5.

40. Matthews T, Boehme R, Antiviral activity and mechanism of action of ganciclovir, Rev Infect Dis, 1988;10 Suppl 3:S490-4.

41. Hamzeh FM, Lietman PS, Gibson W, et al., Identification of the Iytic origin of DNA replication in human cytomegalovirus by a novel approach utilizing ganciclovir-induced chain termination, J Virol, 1990;64:6184-95.

42. Elion GB, The biochemistry and mechanism of action of acyclovir, I Antimicrob Chemother, 1983;12 Suppl B:9-17.

43. Biron KK, Stanat SC, Sorrell JB, et al., Metabolic activation of the nucleoside analog 9-[( 2-hydroxy-1(hydroxymethyl)ethoxy]methyl)guanine in human diploid fibroblasts infected with human cytomegalovirus, Proc Natl Acad SCi U S A, 1985;82:2473-7.

44. St Clair MH, Miller WH, Miller RL, et al., Inhibition of cellular alpha DNA polymerase and herpes simplex virus-induced DNA polymerases by the triphosphate of BW759U, Antimicrob Agents Chemother, 1984:25:191-4.

45. Cheng YC, Huang ES, Lin JC, et al., Unique spectrum of activity of 9-[(1,3-dihydroxy-2-propoxy)methyl]-guanine against herpesviruses in vitro and its mode of action against herpes simplex virus type 1, Proc Natl Acad Sci U S A, 1983b;80:2767-70.
46. Athmanathan S, Garg P, Rao GN, Ophthalmic antiviral chemotherapy: an overview, Indian J ophthalmol 1997;45:203-10.

47. Inoue $Y$, Ohashi $Y$, Shimomura $Y$, et al., Comparative efficacy of three antiviral drugs in mice herpetic keratitis, Jpn J ophthalmol, 1989;33:125-31.

48. Shaw MM, Gurr WK, Watts PA, et al., Ganciclovir and penciclovir, but not acyclovir, induce apoptosis in herpes simplex virus thymidine kinase-transformed baby hamster kidney cells, Antivir Chem Chemother, 2001;12:175-86.

49. Shiota H, Naito T, Mimura Y, Anti-herpes simplex virus (HSV) effect of 9-(1,3-dihydroxy-2-propoxymethyl)guanine (DHPG) in rabbit cornea, Curr Eye Res, 1987;6:241-5.

50. Trousdale MD, Nesburn AB, Willey DE, et al., Efficacy of BW759 (9-[[2-hydroxy-1(hydroxymethyl)ethoxy]methyl]guanine) against herpes simplex virus type 1 keratitis in rabbits, Curr Eye Res, 1984;3:1007-15

51. Castela N, Vermerie N, Chast F, et al., Ganciclovir ophthalmic gel in herpes simplex virus rabbit keratitis: intraocular penetration and efficacy, J Ocul Pharmacol, 1994:10:439-51.

52. Tabbara KF, Treatment of herpetic keratitis, Ophthalmology, 2005;112:1640

53. HEDS, Oral acyclovir for herpes simplex virus eye disease: effect on prevention of epithelial keratitis and stromal keratitis. Herpetic Eye Disease Study Group, Arch Ophthalmol, 2000:118:1030-6.

54. Trousdale MD, Goldschmidt PL, Nobrega R, Activity of ganciclovir against human adenovirus type-5 infection in cel culture and cotton rat eyes, Cornea, 1994;13:435-9.

55. Taylor DL, Jeffries DJ, Taylor-Robinson D, et al., The susceptibility of adenovirus infection to the anti-cytomegalovirus drug ganciclovir (DHPG), FEMS Mircobiol Lett, 1998;49:337-41.

56. Tabbara KF, Jarade EF, Ganciclovir effects in adenovira keratoconjunctivitis, IOVS, 2001;42(4):S579.

57. Verin PH, Mortemousque B, Barach D, Ganciclovir $0.15 \%$ gel, a new treatment in epidemic jeratoconjunctivitis (EKC), Ophthalmic Res, 1997:29(Suppl. 1):12-27.

58. Bruno B, Gooley $T$, Hackman RC, et al, Adenovirus infection in hematopoietic stem cell transplantation: effect of ganciclovir and impact on survival, Biol Blood Marrow Transplant, 2003;9:341-52

59. Gordon YJ, Romanowski E, Araullo-Cruz T, et al., Inhibitory effect of (S)-HPMPC, (S)-HPMPA, and 2'-nor-cyclic GMP on clinical ocular adenoviral isolates is serotype-dependent in vitro, Antiviral Res, 1991;16:11-6.

60. Kinchington PR, Romanowski EG, Jerold Gordon Y, Prospects for adenovirus antivirals, J Antimicrob Chemother, 2005;55:424-9.

61. Schonberger S, Meisel R, Adams $\mathrm{O}$, et al., Prospective, comprehensive, and effective viral monitoring in children undergoing allogeneic hematopoietic stem cell transplantation, Biol Blood Marrow Transplant, 2010:16:1428-35.

62. Baker, FDA approves oral ganciclovir as first drug to prevent CMV disease. US FDA National Library of Medicine AIDSline, Beta, 1995.

63. Chee SP, Jap A, Cytomegalovirus anterior uveitis: outcome of treatment, Br J Ophthalmol, 2010:94:1648-52.

64. Chau Tran TH, Cassoux N, Bodaghi B, et al., Successful treatment with combination of systemic antiviral drugs and intravitreal ganciclovir injections in the management of severe necrotizing herpetic retinitis, Ocul Immunol Inflamm, 2003;11:141-4.

65. Kim SJ, Equi R, Belair ML, et al., Long-term preservation of vision in progressive outer retinal necrosis treated with combination antiviral drugs and highly active antiretroviral therapy, Ocul Immunol Inflamm, 2007;15:425-7.

65. Tam PM, Hooper CY, Lightman S, Antiviral selection in the management of acute retinal necrosis, Clin Ophthalmol, 2010:4:11-20. 